\title{
Recent impact study of automatic search function of the pacemaker atrial hysteresis on atrial arrhythmias
}

\author{
Yongdong Li, Zhiping Ge, Huihua Wen, Anjie Xu \\ Department of Cardiology, The Third Affiliated Hospital of Inner Mongolia Medical College, Baotou, China
}

Received: October 10, 2016

DOI: $10.14725 /$ dcc.v3n4p14
Accepted: November 6, $2016 \quad$ Online Published: December 10, 2016

URL: http://dx.doi.org/10.14725/dcc.v3n4p14

\begin{abstract}
Objective: To explore the recent influences of pacemaker with automatic search function of atrial hysteresis on atrial arrhythmias, and to evaluate it clinical efficacy and safety.

Methods: Indentify ADx DDD 5286 implanted dual chamber pacemaker for sick sinus syndrome in 43 cases. Automatic search of atrial lag was not opened with after pacemaker implantation, and the pacemaker settings were kept. Follow-up program after 3 months, DDD mode with automatic search of atrial lag was opened, and this mode was followed up for 6 months, comparing the atrial pacing percentage and DCG atrial tachyarrhythmias of pacemaker implantation to opening atrium lag mode.

Results: Compared with the preoperative and operative 3 months later, dynamic electrocardiogram (DCG) $24 \mathrm{~h}$ showed that the number of atrial premature beats (APB) and atrial tachycardia, atrial fibrillation (AF) array increased $(p<.05)$; the cases of APB, atrial tachycardia and AF episodes were also increased $(p<.05)$. Compared with the automatic search function in atrial hysteresis model opened with and not opened: atrial pacing percentage decreased $[0.54(0.41,0.71)$ vs. $0.82(0.65,0.93), p<$ $.05]$; DCG $24 \mathrm{~h}$ showed that the number of APB, AF episodes was reduced $(p<.05)$.

Conclusions: Automation search function in atrial hysteresis model can obviously reduce the proportion of atrial pacing, reduce the occurrence of atrial arrhythmias; opened with automatic search function in atrial hysteresis model was safe and reliable.
\end{abstract}

Key Words: Pacemaker, Artificial, Arrhythmias, Cardiac, Automatic search, Atrial delayed

In recent years, physicians in the field of pacemaker and electrophysiology have focused their attention on the study of apex pacing of the right ventricle and different ventricular pacing sites. ${ }^{[1]}$ In fact, the right atrial appendage is also a non physiological part of the pacing. The pacing at the same site can also cause abnormal electrical activity of the heart, resulting in the asynchrony of the left and right atrial and their chambers, promoting the reentry of the atrium, and easily inducing reentrant atrial arrhythmias. Secondly, the unnecessary atrial pacing obviously increases the energy consumption. ${ }^{[2,3]}$ Therefore, it is of great significance to reduce the ratio of atrial pacing in clinic. Automatic search of atrial lag can initiatively extend the AA interval regularly, and encourage the occurrence of sinus rhythm, so as to further reduce the purpose of atrial pacing. The paper aims to explore the recent influences of pacemaker with automatic search function of atrial hysteresis on atrial arrhythmias, and to evaluate its clinical efficacy and safety.

\section{Materials and methods}

\subsection{General information}

A total of 43 cases of sick sinus syndrome undergoing Identity Adx DDD 5286 implanted dual chamber pacemaker in the Department of Cardiology of the Third Affiliated Hos-

\footnotetext{
*Correspondence: Yongdong Li; E-mail: lyd20070501@163.com; Address: Department of Cardiology, The Third Affiliated Hospital of Inner Mongolia Medical College, Baotou, China.
} 
pital of Inner Mongolia Medical College, from May 2011 to June 2012, were selected as the study subject. All patients were in accordance with the following conditions. (1) The patients were eligible for the treatment of the dual chamber pacemaker. They were willing to participate in the study and were able to complete the follow-up. (2) The rate of the patient's own atrium was lower than the basic frequency of program control. Exclusion criteria: patients whose own atrium rate was higher than the basic frequency of program control.

\subsection{Methods for the implantation}

The pacing wires were implanted via cephalotomy or subclavian vein puncture. The right ventricular electrode was placed in the apex of the right ventricle, and the right atrium electrode was placed in the right auricle. The atrium and ventricle were all passive electrodes. According to $X$-ray lateral fluoroscopy, electrocardiogram and pacing threshold, impedance, $\mathrm{P}$ wave and $\mathrm{R}$ wave amplitude, the location of electrode leads was determined.

\subsection{Programming control of peacemaker}

Automatic search of atrial lag was not opened with after pacemaker implantation, and the pacemaker settings were kept. Follow-up program after 3 months, DDD mode with automatic search of atrial lag was opened, and this mode was followed up for 6 months. The standard of programming control includes: the basic frequency was 60 times/min, the lag frequency was 50 times/min, the search period was $30 \mathrm{~min}$, and the number of search cycles was 2. Before and after the pacemaker implantation, the atrial lag mode was not opened after 3 months. Then the $24 \mathrm{~h} \mathrm{dy}$ namic electrocardiogram (DCG) was performed at 3 months follow-up. DCG used three channel portable $128 \mathrm{MB}$ flash cards and three channel recorder $(\mathrm{CH} 1, \mathrm{CH} 2, \mathrm{CH} 3)$. The number of $24 \mathrm{~h}$ atrial early times, atrial tachycardia and atrial fibrillation (AF) array were replayed and calculated by computer system. No antiarrhythmic drugs were administered before and after pacemaker implantation.

\subsection{Follow-up visit}

The patient's complaint was recorded during a 6-month follow-up. The percentage of atrial pacing was recorded at each follow-up, and the threshold of the atrium and ventricle, the impedance of the wire, the amplitude of $\mathrm{P}$ wave and $\mathrm{R}$ wave were measured.

\subsection{Statistical analysis}

Statistical analysis was performed using SPSS13.0 software, and represented by $\bar{x} \pm \mathrm{s}$. The comparison between

Published by New Century Science Press groups was made by use of $t$-test. Before and after pacemaker implantation, the number of atrial premature, atrial tachycardia, AF and percentage of atrial pacing were expressed in the median $(25 \%$ percentile and $75 \%$ percentile, $\left[\mathrm{M}\left(\mathrm{P}_{25}, \mathrm{P}_{75}\right)\right]$ before and after pacemaker implantation by nonparametric Mannwhipney test. The detection rate of atrial arrhythmia before and after pacemaker implantation and open atrial lag mode was compared with $\chi^{2}$ square test, with a difference of $p<.05$, indicating a statistically significant difference.

\section{Results}

\subsection{The change of atrial pacing ratio in atrial lag mode}

The atrial pacing mode with automatic search was opened for 3 months, and the pacing percentage of the atria significantly decreased compared with that before opening $[0.54$ $(0.41,0.71)$ vs. $0.82(0.65,0.93), p<.05]$.

\subsection{The onset of atrial arrhythmia before and after the atrial lag mode was opened}

In 43 cases, DCG was recorded before pacemaker implantation. In 6 months follow-up, DCG of 41 cases and 39 cases was recorded in 3 months and 6 months after operation respectively. Compared with preoperative, the number of DCG $24 \mathrm{~h}$ atrial premature, atrial tachycardia and AF was increased $(p<.05)$ in 3 months after operation. Open atrial lag mode was compared with that of unopened pattern. The number of DCG $24 \mathrm{~h}$ atrial premature, atrial tachycardia and AF was significantly decreased (see Table 1). A comparison was made between 39 patients who had completed a 6-month follow-up. Compared with the preoperative condition, atrial tachycardia and AF episodes were increased ( $p$ $<.05)$ in 3 months after the operation. Compared with the unopened model, the number of episodes of atrial premature lag, atrial tachycardia, and AF decreased $(p<.05)$ (see Table 2).

\subsection{The changes in the pacing threshold of the atrium and ventricle, the impedance of the wire, $P$ wave and $R$ wave}

There was no significant change in pacing threshold, the impedance of the wire, $\mathrm{P}$ wave and $\mathrm{R}$ wave amplitude in 6 months follow-up, $p>.05$ (see Table 3 ).

2.4 The clinical manifestation of the patients before and after the atrial lag mode was opened

There was no discomfort in patients with opened and unopened atrial lag model. 
Table 1: The onset of DCG atrial arrhythmia before and after pacemaker implantation, and after the atrial lag mode was opened [Times/24 h, M $\left(\mathrm{P}_{25}, \mathrm{P}_{75}\right)$ ]

\begin{tabular}{lllll}
\hline Time & Cases & Atrial premature beats & Atrial tachycardia & Atrial fibrillation \\
\hline Preoperative & 43 & $140.00(0.00,1,472.00)$ & $13.00(1.00,112.00)$ & $2.00(1.00,5.00)$ \\
Operative 3 months later & 41 & $278.00(0.00,1986.00)^{\#}$ & $24.00(1.00,98.00)^{\#}$ & $4.00(1.00,9.00)^{\#}$ \\
Operative 6 months later & 39 & $8.00(0.00,91.00)^{*}$ & $1.00(1.00,7.00)^{*}$ & $0.00(0.00,0.00)^{*}$ \\
\hline
\end{tabular}

Note. Compared with operative 3 months later, ${ }^{*} p<.05$; Compared with preoperative, ${ }^{\#} p<.05$

Table 2: The rate of pacemaker implantation, atrial arrhythmia before and after the atrial lag mode was opened (Cases, \%)

\begin{tabular}{llll}
\hline Time & Atrial premature beats & Atrial tachycardia & Atrial fibrillation \\
\hline Preoperative & $14(35.9)$ & $9(23.1)$ & $4(33.3)$ \\
Operative 3 months later & $22(56.4)^{\#}$ & $17(43.6)^{\#}$ & $9(23.1)^{\#}$ \\
Operative 6 months later & $10(25.6)^{*}$ & $6(15.4)^{*}$ & $1(33.3)^{*}$ \\
\hline
\end{tabular}

Note. Compared with operative 3 months later, ${ }^{*} p<.05$; Compared with preoperative, ${ }^{\#} p<.05$

Table 3: The changes in the pacing threshold of the atrium and ventricle, the impedance of the wire, $\mathrm{P}$ wave and $\mathrm{R}$ wave before and after pacemaker implantation, and after the atrial lag mode was opened $(\bar{x} \pm \mathrm{s})$

\begin{tabular}{|c|c|c|c|c|c|c|c|}
\hline \multirow{2}{*}{ Time } & \multirow{2}{*}{ Cases } & \multicolumn{2}{|c|}{ Atrial premature beats } & \multicolumn{2}{|c|}{ Atrial tachycardia } & \multicolumn{2}{|c|}{ Atrial fibrillation } \\
\hline & & Atrium & Ventricle & Atrium & Ventricle & Atrium & Ventricle \\
\hline Preoperative & 43 & $0.91 \pm 0.27$ & $0.57 \pm 0.21$ & $2.17 \pm 0.64$ & $7.58 \pm 0.57$ & $518 \pm 119$ & $637 \pm 121$ \\
\hline Operative 3 months later & 41 & $0.87 \pm 0.20$ & $0.59 \pm 0.18$ & $2.07 \pm 0.84$ & $7.54 \pm 0.54$ & $524 \pm 120$ & $634 \pm 127$ \\
\hline Operative 6 months later & 39 & $0.89 \pm 0.21$ & $0.58 \pm 0.20$ & $2.19 \pm 0.50$ & $7.56 \pm 0.68$ & $521 \pm 113$ & $629 \pm 118$ \\
\hline
\end{tabular}

\section{Discussion}

The right atrial appendage is located at the anterior part of the right atrium. Most of them have deep trabecular, forming a natural pocket, which is conducive to placement and fixation of electrodes, and thus become the traditional part of atrial pacing. However, the electrophysiological defects in the right atrial pacing also prolong the time of the room conduction. In that case, left atrial activation is later than right atrial activation, thus causing out-sync of left and right atrial, increasing of $\mathrm{P}$ duration and $\mathrm{P}$ wave dispersion, which is easy to cause the occurrence of reentrant atrial arrhythmias. ${ }^{[4]}$ In addition, unnecessary atrial pacing obviously increases energy consumption. In theory, the above disadvantages can not be avoided as long as it is not paced on the normal conduction beam. ${ }^{[5]}$ Obviously, it is essential clinically to reduce the rate of atrial pacing.

Similar to reducing atrioventricular automatic search function of ventricular pacing, the atrial frequency hysteresis function with search can regularly extend AA interval to search and encourage the occurrence of sinus rhythm, and further achieve the purpose of atrial pacing. ${ }^{[6,7]}$ The Identity Adx DDD 5286, a product of St. jude's dual chamber pacemaker, can actively prolong VA interval, that is, AF with automatic search function search for sinus rhythm. If the sinus rhythm is detected, the VA interval will work with the programmed frequency. It increases the active search function on the basis of starting the lag frequency after the traditional perception of its own $\mathrm{P}$ wave, and the time of search is programmable. The interval of pacing can be prolonged regularly and the heart rate can be searched. If the $P$ wave is still absent after the delay frequency interval (VA interval prolongation), the pacemaker will automatically restore the VA interval to the basic frequency interval. This study showed that the number of atrial premature, atrial tachycardia and AF increased at DCG 24 h 3 months after surgery. The number of episodes of atrial premature, atrial tachycardia and atrial fibrillation increased $(p<.05)$, indicating that too much right atrial appendage pacing is likely to induce reentrant atrial arrhythmia, which is consistent with previous reports. ${ }^{[2]}$ The atrial lag was opened with automatic search for 3 months, and the rate of pacing in the anterior atrium was significantly lower than that in the anterior atrium. At the same time, the comparison between the mode of opening and unopened atrial lag was made. The number of DCG $24 \mathrm{~h}$ atrial early times, atrial tachycardia and AF decreased significantly. The number of episodes of onset of atrial lag, atrial tachycardia, and atrial fibrillation was sig- 
nificantly reduced. Therefore, it is suggested that opening atrial lag mode to reduce atrial pacing ratio has preventive effect on atrial premature, atrial tachycardia and atrial fibrillation. The possible mechanism may be related to the effect of the right atrial appendage on the asynchrony of the left and right atrium that promotes atrial reentry. Opening atrial lag mode improves the incidence of dyssynchrony with decreasing atrial pacing ratio, thus reducing the incidence of atrial arrhythmia. ${ }^{[7-10]}$

During the follow-up, no symptoms of heart palpitations, shortness of breath, syncope, amaurosis or any other discomfort were described, suggesting the safe and reliable use of pacemakers with auto search atrial hysteresis model.

\section{References}

[1] Kolb C, Schmidt R, Dietl JU, et al. Reduction of right ventricular pacing with advanced atrioventricular search hysteresis: results of the PREVENT study. Pacing Clin Electrophysiol. 2011; 34: 975-983. PMid: 21438891. https://doi.org/10.1111/j. $1540-8159.2011 .03075 . x$

[2] Xu JF, Su YG. Correct understanding of atrial septum pacing. Chinese Journal of Cardiac Pacing and Electrophysiology. 2008; 22: 287.

[3] Wang XD, Wang YM. The modern concept of physiological pacing. Advances in Cardiovascular Disease. 2009; 32: 249-254.

[4] Strohmer B, Pichler M, Froemmel M, et al. Evaluation of atrial conduction time at various sites of right atrial pacing an influence on atrioventricular delay optimization by surface electocardiology. PACE. 2004; 27: 468-474. PMid: 15078399. https://doi.org/ $10.1111 / j .1540-8159.2004 .00465 . x$
Therefore, patients with sick sinus syndrome with implanted pacemaker indications are suggested to choose pacemakers with automatic atrial lag function when their atrial rate is lower than the basic frequency of program.

The limitation of this study is that only small sample in a single center is involved, and the monitoring time is relatively short. Multi center, large sample clinical trials and long-term follow-up studies still need to be evaluated in the future.

\section{Conflicts of Interest Disclosure}

The authors have no conflicts of interest related to this article.

[5] Chen X. Clinical arrhythmia ( $2^{\text {nd }}$ edition). Beijing: People's Health Press; 2009. 1096-1099 p.

[6] Melzer C, Sowelam S, Sheldon TJ, et al. Reduction of right ventricular pacing in patients with sinus node dysfunction using an enhanced search AV algorithm. Pacing Clin Electrophysiol. 2005; 28: 521-527. PMid: 15955184. https://doi.org/10.1111/j. 1540-8159.2005.09470.x

[7] Su YG, Geng JB. Analysis of new function of cardiac pacemaker. Shanghai: Shanghai Science and Technology Press; 2009. 60-66 p.

[8] Wang Y, Zhang R, Wang X, et al. The effect of high right ventricular septum pacing VAT and DDD on left ventricular function in patients with third degree atrioventricular block. Chinese Journal of Cardiac Pacing and Electrophysiology. 2012; 26: 33-35.

[9] Li T, Wu Q. Clinical study on the prevention and treatment of atrial fibrillation by Adapta pacemaker. International Journal of Cardiovascular Disease. 2012; 393: 189-190.

[10] Zhang Y, Ren XQ, Wang FZ. Standardized follow-up and program control after cardiac pacemaker implantation. Molecular Cardiology of China. 2012; 12:147-149. 